\title{
Theoretical Analysis of the Relative Impact of Obesity on Hemodynamic Stability During Acute Hemorrhagic Shock
}

\author{
Sarah A. Sterling ${ }^{1}$; Alan E. Jones ${ }^{1}$; Thomas G. Coleman ${ }^{1}$; Richard L. Summers ${ }^{1, *}$ \\ ${ }^{1}$ Department of Emergency Medicine, University of Mississippi Medical Center, Jackson, Mississippi, USA \\ ${ }^{*}$ Corresponding author: Richard L. Summers, Department of Emergency Medicine, University of Mississippi Medical Center, Jackson, Mississippi, USA. Tel: +1601-8158245; \\ +1601-9845583, E-mail: rsummers@umc.edu
}

Received: August 7, 2014; Revised: March 16, 2015; Accepted: June 16, 2015

\begin{abstract}
Background: Evidence suggests that morbid obesity may be an independent risk factor for adverse outcomes in patients with traumatic injuries.

Objectives: In this study, a theoretic analysis using a derivation of the Guyton model of cardiovascular physiology examines the expected impact of obesity on hemodynamic changes in Mean Arterial Pressure (MAP) and Cardiac Output(CO) during Hemorrhagic Shock (HS). Patients and Methods: Computer simulation studies were used to predict the relative impact of increasing Body Mass Index (BMI) on global hemodynamic parameters during HS. The analytic procedure involved recreating physiologic conditions associated with changing BMI for a virtual subject in an In Silico environment. The model was validated for the known effect of a BMI of 30 on iliofemoral venous pressures. Then, the relative effect of changing BMI on the outcome of target cardiovascular parameters was examined during simulated acute loss of blood volume in class II hemorrhage. The percent changes in these parameters were compared between the virtual nonobese and obese subjects. Model parameter values are derived from known population distributions, producing simulation outputs that can be used in a deductive systems analysis assessment rather than traditional frequentist statistical methodologies.

Results: In hemorrhage simulation, moderate increases in BMI were found to produce greater decreases in MAP and CO compared to the normal subject. During HS, the virtual obese subject had $42 \%$ and $44 \%$ greater falls in CO and MAP, respectively, compared to the nonobese subject. Systems analysis of the model revealed that an increase in resistance to venous return due to changes in intra-abdominal pressure resulting from obesity was the critical mechanism responsible for the differences.

Conclusions: This study suggests that obese patients in HS may have a higher risk of hemodynamic instability compared to their nonobese counterparts primarily due to obesity-induced increases in intra-abdominal pressure resulting in reduced venous return.
\end{abstract}

Keywords: Shock; Hemorrhagic; Obesity; Trauma; Hemodynamics

\section{Background}

According to recent national estimates, over 78 million adults and 12.5 million children and adolescents in the United States are obese (1). Despite the remarkable prevalence of obesity in the U.S. and the many known detrimental health effects caused by obesity, there are still a number of mysteries surrounding the effects of obesity on both therapeutic and diagnostic care, particularly in the area of trauma. Previous research has suggested that obese patients may be at greater risk for increased morbidity and mortality, though these findings have not been entirely consistent (2-5). There have been many proposed etiologies for the differences in outcomes in obese trauma patients. A recent, retrospective study by Nelson and colleagues found that obese patients were at increased risk for early hypovolemic shock and higher subsequent mortality (6). They did note, however, that when correcting for Body Mass Index (BMI), obese patients received significantly smaller fluid volumes during the initial resuscitation period, creating an obvious potential confounder (6). Similarly though, animal studies have dem- onstrated that obese rats have impaired hemorrhage tolerance compared to their nonobese counterparts $(7,8)$. Noting this compelling, though limited evidence, we sought to further examine the effects of obesity in hemorrhagic shock using a systems analysis approach. Computational modeling is a validated methodology for the evaluation and analysis of clinically relevant questions that might be feasibly difficult to answer (9-11). In trauma patients, particularly those with significant hemorrhage, therapeutic treatments are time sensitive, and fully evaluating all potential effects of obesity on cardiovascular physiology could be difficult and potentially complicate resuscitations without preliminary data. Additionally, the modeling and systems analytic approach allows for a more thorough examination of the mechanisms responsible for the observed phenomena. The Guyton model of cardiovascular physiology is a well-studied and previously published means for evaluating circulatory functioning, and the anticipated effects of changes on the cardiovascular system (12-15). Continued expansion

Copyright (C) 2015, Kashan University of Medical Sciences. This is an open-access article distributed under the terms of the Creative Commons Attribution-NonCommercial 4.0 International License (http://creativecommons.org/licenses/by-nc/4.0/) which permits copy and redistribute the material just in noncommercial usages, provided the original work is properly cited. 
of the model to over 5000 individual physiological variables also includes integrated functioning of neuroendocrine and metabolic systems in circulatory control. Recent analysis has found that the extended version of the Guyton model is able to accurately predict physiologic responses to hemorrhage $(16,17)$.

\section{Objectives}

The objective of this study was to perform a theoretic analysis using a derivation of the Guyton model of cardiovascular physiology to evaluate the impact of obesity on expected hemodynamic changes in Mean Arterial Pressure (MAP) and Cardiac Output (CO) during the very early stages (the golden hour of trauma) of acute Hemorrhagic Shock (HS) and examine the possible physiologic mechanisms responsible for these changes.

\section{Patients and Methods}

The methods used in this study were those required for a theoretic analysis of the effects of increasing BMI on the global hemodynamics changes during hemorrhage. The analytic procedure involved recreating the physiologic conditions associated with changing BMI for a virtual subject created with the use of a mathematical model of human physiology. In keeping with the national heart, lung, and blood institute's terminology, we considered a BMI of $20-24.9 \mathrm{mg} / \mathrm{kg}^{2}$ as normal weight and a BMI of $\geq 30 \mathrm{mg} / \mathrm{kg}^{2}$ as Grade I obesity (18). First, the model was validated for the known effect of a BMI of 30 on iliofemoral venous pressures. Then, the relative effect of changing BMI on the outcomes of target cardiovascular parameters (cardiac output and mean arterial between the virtual normal weight and obese subjects. Given that no human or animal subjects were involved in this study, an institutional review was not required.

\subsection{Computational Platform and Systems Analysis Protocol}

The computational methodology used in the systems analysis employs a well-established computer model of human physiology (Guyton/Coleman/Summers model). This model has been developed over the past 30 years and describes the integrative cardiovascular physiologic functioning of a virtual subject $(10,12,13,15)$. The mathematical package used in the current analysis was a homegrown product developed by one of the authors (Coleman) using $\mathrm{C}++$ as the programming language. This model and methodology, which previously have been used in numerous studies, are intended to provide a more detailed understanding of the physiologic mechanisms involved in common clinical conditions $(11,13-15,19,20)$. Further, several versions of this model have been previously demonstrated to accurately predict hemodynamic changes seen during hypotensive states $(11,17,20)$. The model has been specifically validated previously for hemorrhagic states in a published study in which humans were placed in a simulated shock state by a standard lower body negative pressure technique (17). In this paper the model is further validated for the state of obesity as described below. While it is not expected that there will be absolute agreement between the model output and experimental findings, there should be definite measurable criteria by which to judge the validity of the model $(9,17)$. Specific methods and criteria previously described in the literature were used in the validation process of the computational platform and are presented and referenced in our previous papers $(9,17)$. The overall dynamic performance of the model was quantified by determination of the median performance error (MDPE $=$ median $[\mathrm{PE}]$ over all data points as $\%$ of measured where $\mathrm{PE}=$ difference between measured and predicted values). MDPE has been considered as the measure of the bias and inaccuracy between the model's predictions and the corresponding experimental observations. This measure has been employed in the validation of algorithms used in insulin pumps and drug delivery systems in which precision is of the utmost of clinical importance (17). A commonly considered error margin of $5 \%$ was chosen a priori for this study.

This evaluation suggests that the model can be used as a platform for the theoretical analysis of such shock states. The model contains numerous parameters that describe the detailed interactions of systemic, organ, and tissue cellular physiology and metabolism using basic physical principles and established biologic relationships. The model's structure not only incorporates the cardiovascular and neurogenic physiologic responses to changes in pressures, flows, and hydraulics within the circulatory system, but also incorporates the utilization and mass balance fluctuations of metabolic substrates. The details of this model structure are beyond the scope of the current paper though they have been described in previous publications $(12,13)$.

The systems analytic procedure using the computational platform involves recreating the clinical scenario for virtual obese and nonobese subjects within an In Silico environment $(17,18)$. We performed a series of simulation studies in which the states of truncal obesity and nonobesity were instantiated within the model parameter sets with their concomitant impacts on vascular, metabolic, and endocrine components. Two of the major physiologic elements included in this state change were a shift in the insulin/glucagon axis secondary to changes in receptor sensitivities and an increase in the intraabdominal pressures from a normal range of $5 \mathrm{mmHg}$ in the nonobese to a level of $15 \mathrm{mmHg}$ in the obese (21-26). Both of these pathophysiologic changes have been documented in clinical studies in human subjects. The computer simulation was allowed to run until the model system blood pressure reached a steady state. The determined values for the cardiac outputs and mean arterial pressures, as driving hemodynamic components of shock, were then compared for the virtual obese and nonobese individuals. 
A clearer understanding of the basic model functioning and dynamics can be obtained using a graphical analysis of cardiac output control as was developed by Dr. Arthur C. Guyton, one of the progenitors of the current model (Figure 1). The cascade of hemodynamic and physiologic regulatory responses seen during an acute hemorrhagic event can be succinctly illustrated from an analysis of a classic Guyton diagram of the control of cardiac output and venous return. The hemodynamics of blood flow in the circulation can be considered dynamically as an intersection between the curves for cardiac output as determined by the Starling functional relationship to atrial pressures and cardiac filling and the impact of those same factors for influencing venous return. The equilibrium intersection of those curves (point $\mathrm{A}$ ) determines the blood flow. With hemorrhage, the loss of volume in the circulation immediately results in a concurrent reduction in the Mean Circulatory Filling Pressure (MCFP) which results in a loss of drive for venous return and cardiac filling. This is depicted by a shift in the venous return curve to the left (\#1) and a new equilibrium (point B). The reduction in venous return and subsequent diminished cardiac output elicits a counter regulatory acute response of increase neurohormonal output including sympathetic drive, norepinephrine, epinephrine, renin-angiotensin activation, and vasopressin release. Short-term and longterm arterial pressure control mechanisms are also in play within the model including vascular autoregulation and stress-relaxation responses. These are all demonstrated by a shift in the venous return curve to the right (\#2) with a new equilibrium maintaining flow under stressed conditions (point C). If cardiac functions begin to diminished under a sustained stressed without timely blood volume resuscitation (\#3), then flow will also begin to deteriorate especially if the hemorrhage is ongoing and there is further loss in venous return (Points D to E). A vicious cycle can ensue with further suppression of cardiac function, diminished flow and eventual irreversible circulatory collapse. These physiological dynamics are all central to the functioning of the model.

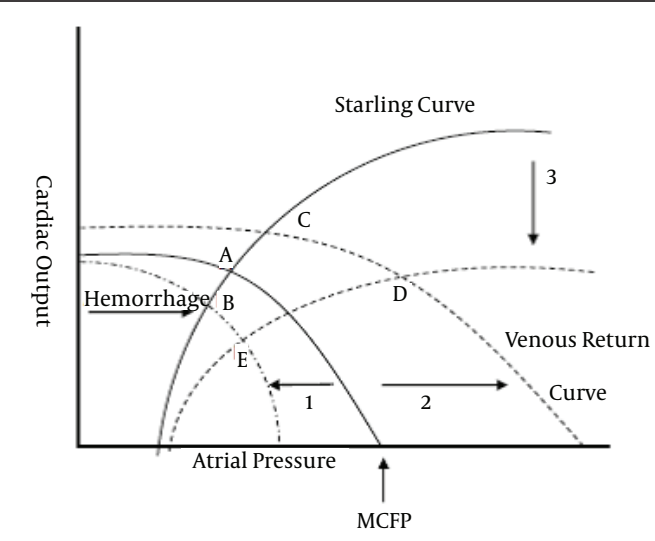

Figure 1. Graphical Analysis of Cardiac Output as Determined by the Intersection Between the Starling and the Venous Return Curves
The equilibrium point (A) defines the steady state cardiac output at a specific atrial pressure. The venous return (and cardiac output) becomes zero when the atrial pressure equals the MCFP. With the initial hemorrhage the MCFP is reduced and the venous return curve shifts to the left (1) to form a new equilibrium point (B) with reduced cardiac output. Neurohormonal responses attempt to bring the curve back to the right (2) to achieve a stable equilibrium point $(C)$. If the hemorrhaged state persists without resuscitation then a depression of the cardiac starling curve can ensue (3) and lower cardiac output again. If the hemorrhage progresses a vicious cycle can result in a shock state with a low cardiac output and low atrial pressure (E).

\subsection{Model Validation for Obesity}

While there has been extensive validation and documentation of the basic computational model used in this study, to assure credibility in this context, the relevant elements of the model predictions for the obese patient were compared to experimental data from the literature. Although it is unrealistic to expect a generalized computational model to exactly reproduce the experimental results of select study participants, the observed results of these baseline simulations should accurately reflect the direction and degree of expected physiologic changes as set by standard methods used for validations of similar analyses (9-11). The target simulation experiment used in this validation differentiated the obese from nonobese subject while tracking the changes in venous pressures distal to the vena cava bifurcation.

1. According to literature sources, the distal inferior vena cava pressure increases by about $125 \%$ of baseline values when the abdominal pressure of a normal individual is increased through abdominal cuff compression methods to $20 \mathrm{mmHg}$ (25). In the simulation, the venous pressure increases by $108 \%$ with the same increase in external abdominal pressure which is a $17 \%$ difference.

2. Experimental evidence indicates that morbidly obese individuals with estimated intra-abdominal pressures of about $14 \mathrm{mmHg}$ were found to have femoral venous pressures of about $15 \mathrm{mmHg}(26)$. In the simulation, the pressure rises to $14 \mathrm{mmHg}$ with the same increase in abdominal pressure, which represents only a $1 \%$ difference.

\section{Results}

During hemorrhage simulation, a moderate increase in the BMI was noted to affect both $\mathrm{CO}$ and MAP. The virtual obese subject had a 31\% decrease in CO and a 23\% decrease in MAP during the class II hemorrhage simulation, while the normal weight patient had only a $22 \%$ and $16 \%$ decrease in $\mathrm{CO}$ and MAP, respectively. The virtual obese subject had a $42 \%$ greater fall in CO than the normal weight virtual patient, and a $44 \%$ greater fall in MAP than the normal weight virtual patient (Figures 2 and 3). 


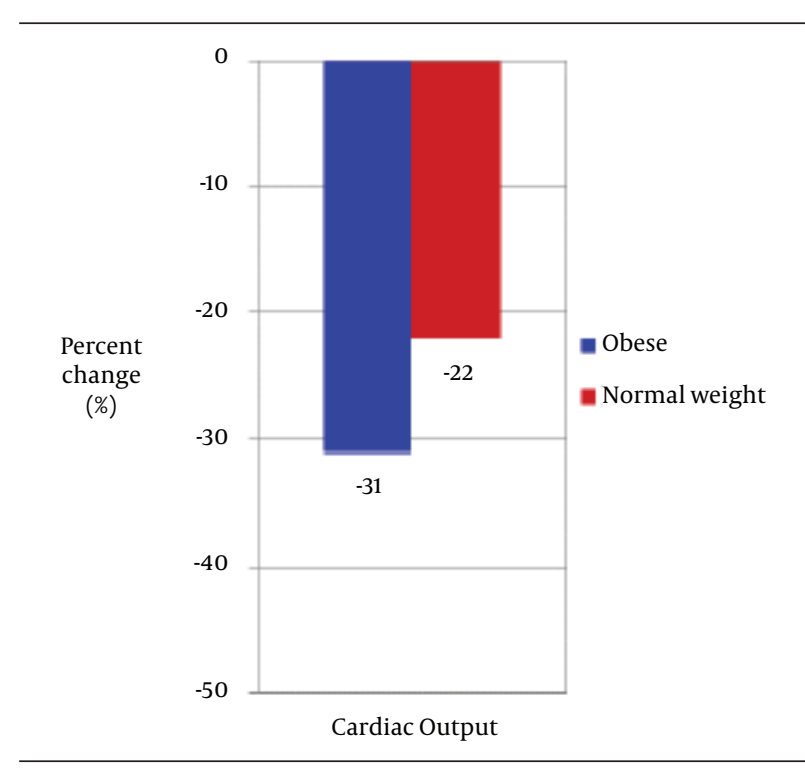

Figure 2. Change in Cardiac Output During Class II Hemorrhage

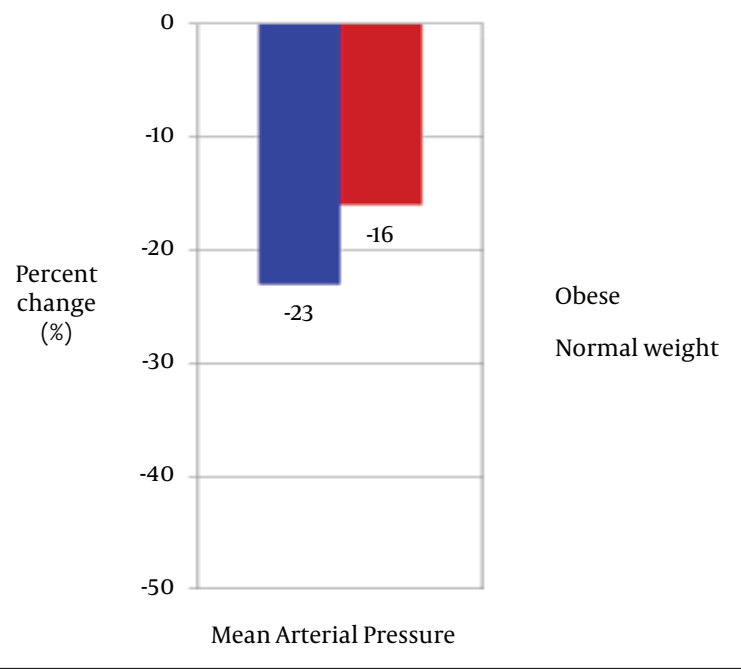

Figure 3. Change in Mean Arterial Pressure During Class II Hemorrhage

To evaluate the etiology of these effects, a systems analysis of the model was performed. This revealed that the critical dominant mechanism for these hemodynamic differences was an increase in the resistance to venous return resulting from an increase in intra-abdominal pressure due to obesity.

\section{Discussion}

In this study of the theoretical effects of obesity in hemorrhagic shock, we found that obese patients are at higher risk for hemodynamic instability with decreased tolerance to volume loss in trauma. These findings could have important clinical practice implications for the management of these patients. As previously mentioned, many studies have found that obese patients are at increased risk for higher morbidity and mortality $(2-4,27)$.
Additionally, recent research has suggested that obese patients may be at higher risk for early hypovolemic shock, but noted that this could be due to relative underresuscitation when correcting for BMI (6).

Our results expand these findings and suggest a physiological source for impaired hemorrhage tolerance in obese individuals due to increased intra-abdominal pressures which in turn increased resistances to venous return. This finding is significant not only in identifying potentially high risk patients, but also by identifying potential avenues for treatment interventions, which could be effective in preventing sustained shock and consequently decreasing morbidity and mortality. For example, in trauma resuscitations in pregnant patients, a left lateral tilt is recommended to improve cardiac output, due to the increased pressure on the venocaval system from the gravid uterus $(28,29)$. Extrapolating from trauma in pregnancy, perhaps simple repositioning of the obese patient could decrease intraabdominal pressures and increase venous return, thereby improving cardiac output. However, unlike our previous study that examined the impact of a uniformly growing gravid uterus on hemorrhagic shock states, a condition of obesity is less homogenous and the influence on the intra-abdominal pressure less readily tracked with changes in size or weight (11). Therefore, a single weight point signifying the lower end of the definition of obesity (BMI 30) was chosen for the current analysis. Likewise, in the pregnancy study we were able to clearly examine the effects of initiating a tilt and gravitational unloading of the gravid uterus on venous return because of its consistent location and influence of a specific area of the vena cava. A larger finite element model would be required to truly examine the impact of the broader regional changes in adipose tissues and organs such as the liver on the abdominal vasculature which is currently beyond the scope of our model.

There is some evidence suggesting that obese patients are at high risk for hypovolemic shock simply due to relative under-resuscitation may also be reflective of this physiologic derangement (6). Some insight into the reasons for this increased risk in the obese trauma patient may be found in the results of our systems analysis. Venous return is a function of the MCFP and resistance to venous return (12). If the resistance is increased this will necessitate an increase in the MCFP to normalize the venous return and cardiac output. This might inform our treatment strategy. A relatively more aggressive fluid resuscitation may be required in the obese as compared to normal weight subjects in order to increase the MCFP and overcome the increased resistance to venous return.

While the focus of this investigation was on the basic plumbing of the hemodynamics in the very early stages of hemorrhagic shock (the golden hour), the model is also capable of exploring metabolic and hormonal responses. The impact of the shifting insulin/glucagon axis on blood pressure is thought to be due to its impacts on renal functioning, sodium balance, and vascular reactiv- 
ity. The possible quantitative role of this axis in arterial pressure control was described through use of a mathematical model in an earlier publication (30). These factors are inherent in the current model and are a part of the 5000 interacting variables. In the acute period (golden hour of trauma) these factors play less of a dominant role as compared to the basic physics of the circulatory hemodynamics and are more relevant during the more protracted timeframes of this type of shock. Heart rate is one of the variables that could have been very easily included in this analysis, but in these early stages it may be less informative due to fluctuating stress related influences that are difficult to model. Therefore there was concern that any simulation driven conclusions about heart rate changes might be misleading.

Further, we found that a modest increase in BMI imparted notable effects on CO and MAP. We compared a virtual normal weight and obese patient in this analysis, with the obese patient defined as a BMI of $30 \mathrm{mg} / \mathrm{kg}^{2}$. The effects of grade II or III obesity (a BMI of $35-39.9 \mathrm{mg} / \mathrm{kg}^{2}$ and $\geq 40 \mathrm{mg} / \mathrm{kg}^{2}$, respectively) could potentially impart even more significant deleterious effects on CO and MAP (18). In this study, however, we used a more conservative cutoff to determine if even moderate increases in BMI could potentially impact physiological response.

The limitations of a computational, theoretical method are evident and involve the inherent assumptions of the mathematical model from both a physiologic and an anatomic position. However, the validation comparisons are considered within the reasonable range of error for this type of biologic modeling (31,32). Further, although more accurate results are possible through manipulation of the mathematics of the model's parameters and structure, this process would not be true to the experimental literature upon which the model was constructed, potentially leading to conclusion with tenuous foundations. The current model has limitations in its degree of anatomic compartmentalization beyond the level of the major organ systems. While this probably does not have a major differential impact on the hydraulics and hemodynamic of acute shock, it may impact the some of the time constants for metabolic exchanges such as oxygen and glucose in this timeframe. However, the limitations of such variables are not evident in the outcomes as compared to the validation measures (17). There are also limitations in the immunologic responses embedded in this model but those factors probably have less impact on our central hypothesis that examines the acute phase. The hormonal and neurologic responses are considerably robust in this model and while there is always room for additional detail, we believe the current features are adequate for the question at hand.

While our simulations suggest that there are substantial differences in the responses of obese and nonobese hemorrhaged patients, there are no statistical determinations to establish the significance of these differences. Unlike clinical studies, in which each point describes a mean outcome for the populations with an associated standard deviation, In Silico studies use methods that are deterministic and characterize the outcomes for a single individual who is representative of the whole population. This approach is considered valid, however, because the separate components of the model's physiology are constructed from population means (11). It may be possible to incorporate the specifics of an individual's physiology into the model so that it can be used for real-time clinical predictions.

The simulations are for only one simple case and do not cover the dynamic spectrum of and ranges of possible obesity and hemorrhage. This approach might be considered to limit the usefulness of the results. We wanted to evaluate the idea at the lowest level of what is clinically identified as obesity and at a modest level of hemorrhage. It was thought that morbid obesity and severe hemorrhage would amplify the results and be misleading. In addition, the occurrence of those conditions combined (morbid obesity and severe hemorrhage) are much less common and therefore would have limited practical clinical relevance.

In simulated Class II hemorrhagic shock, we found that obese subjects had greater decreases in CO and MAP compared to their normal weight counterparts, placing them at higher risk for hemodynamic instability and shock. In this analysis, the primary mechanism for this difference was an increase in intra-abdominal pressure due to obesity, which resulted in increased resistances to venous return. After establishing the theoretical consistency of this primary hypothesis of the impact of obesity on shock in hemorrhage and identifying a probable mechanism through the systems analysis approach, we would hope that future investigations might examine some specifics of time dependent dynamics, derangements of metabolism, treatment schemes and even outcomes in both computer simulations and actual live clinical studies.

\section{Acknowledgements}

Dr. Sterling received support from NIH T32HL105324-03.

\section{Authors' Contributions}

Richard L. Summers and Thomas G. Coleman conceived and carried out experiments. Richard L. Summers and Alan E. Jones analyzed and interpreted the data. Sarah A. Sterling conducted the literature search and generated the figures. All authors contributed in the writing of the manuscript and had final approval of the submitted and published version.

\section{References}

1. Ogden CL, Carroll MD, Kit BK, Flegal KM. Prevalence of obesity in the United States, 2009-2010. NCHS Data Brief. 2012;(82):1-8.

2. Ryb GE, Dischinger PC. Injury severity and outcome of overweight and obese patients after vehicular trauma: a crash in- 
jury research and engineering network (CIREN) study. J Trauma. 2008;64(2):406-11.

3. Neville AL, Brown CV, Weng J, Demetriades D, Velmahos GC. Obesity is an independent risk factor of mortality in severely injured blunt trauma patients. Arch Surg. 2004;139(9):983-7.

4. Arbabi S, Wahl WL, Hemmila MR, Kohoyda-Inglis C, Taheri PA, Wang SC. The cushion effect. J Trauma. 2003;54(6):1090-3.

5. Diaz JJ, Norris PR, Collier BR, Berkes MB, Ozdas A, May AK, et al. Morbid obesity is not a risk factor for mortality in critically ill trauma patients. J Trauma. 2009;66(1):226-31.

6. Nelson J, Billeter AT, Seifert B, Neuhaus V, Trentz O, Hofer CK, et al. Obese trauma patients are at increased risk of early hypovolemic shock: a retrospective cohort analysis of 1,084 severely injured patients. Crit Care. 2012;16(3):R77.

7. Xiang L, Clemmer JS, Lu S, Mittwede PN. Impaired blood pressure compensation following hemorrhage in conscious obese Zucker rats. Life Sci. 2013;93(5-6):214-9.

8. Frisbee JC. Impaired hemorrhage tolerance in the obese Zucker rat model of metabolic syndrome. J Appl Physiol (1985). 2006;100(2):465-73.

9. Summers RL. Computer simulation studies and the scientific method.J Appl Anim Welf Sci. 1998;1(2):119-31.

10. Guyton AC, Montani JP, Hall JE, Manning RJ. Computer models for designing hypertension experiments and studying concepts. Am J Med Sci. 1988;295(4):320-6.

11. Summers RL, Harrison JM, Thompson JR, Porter J, Coleman TG. Theoretical analysis of the effect of positioning on hemodynamic stability during pregnancy.Acad Emerg Med. 2011;18(10):1094-8.

12. Guyton AC, Coleman TG, Granger HJ. Circulation: overall regulation. Annu Rev Physiol. 1972;34:13-46.

13. Guyton AC, Coleman TG. Quantitative analysis of the pathophysiology of hypertension. 1969. J Am Soc Nephrol.1999;10(10):2248-58.

14. Coleman TG. A mathematical model of the human body in health, disease, and during treatment. ISA Trans. 1979;18(3):65-73.

15. Coleman TG, Summers RL. Using mathematical models to better understand integrative physiology.JPhysiol Biochem.1997;53:45-6.

16. Nguyen CN, Simanski O, Kahler R, Schubert A, Janda M, Bajorat J, et al. The benefits of using Guyton's model in a hypotensive control system. Comput Methods Programs Biomed. 2008;89(2):153-61.

17. Summers RL, Ward KR, Witten T, Convertino VA, Ryan KL, Coleman TG, et al. Validation of a computational platform for the analysis of the physiologic mechanisms of a human experimental model of hemorrhage. Resuscitation. 2009;80(12):1405-10.

18. Clinical guidelines on the identification, evaluation, and treatment of overweight and obesity in adults: executive summary. Expert Panel on the Identification, Evaluation, and Treatment of
Overweight in Adults. Am J Clin Nutr. 1998;68(4):899-917.

19. Summers RL, Montani JP, Woodward LH, Coleman TG, Hall JE. Theoretical analysis of the mechanisms of chronic hyperinsulinemia. Comput Biol Med.1997;27(3):249-56

20. Summers RL, Baker SD, Sterling SA, Porter JM, Jones AE. Char acterization of the spectrum of hemodynamic profiles in trauma patients with acute neurogenic shock. J Crit Care. 2013 28(4):531 e1-5.

21. De Keulenaer BL, De Waele JJ, Powell B, Malbrain ML. What is nor mal intra-abdominal pressure and how is it affected by positioning, body mass and positive end-expiratory pressure? Intensive Care Med. 2009;35(6):969-76.

22. Sanchez NC, Tenofsky PL, Dort JM, Shen LY, Helmer SD, Smith RS. What is normal intra-abdominal pressure? Am Surg. 2001;67(3):243-8

23. Nguyen NT, Lee SL, Anderson JT, Palmer LS, Canet F, Wolfe BM Evaluation of intra-abdominal pressure after laparoscopic and open gastric bypass. Obes Surg. 2001;11(1):40-5.

24. Nguyen NT, Wolfe BM. The physiologic effects of pneumoperitoneum in the morbidly obese. Ann Surg. 2005;241(2):219-26.

25. Willenberg T, Clemens R, Haegeli LM, Amann-Vesti B, Baumgartner I, Husmann M. The influence of abdominal pressure on lower extremity venous pressure and hemodynamics: a human in-vivo model simulating the effect of abdominal obesity. Eur Vasc Endovasc Surg. 2011;41(6):849-55.

26. Arfvidsson B, Eklof B, Balfour J. Iliofemoral venous pressure correlates with intraabdominal pressure in morbidly obese patients. Vasc Endovascular Surg. 2005;39(6):505-9.

27. Brown CV, Neville AL, Rhee P, Salim A, Velmahos GC, Demetriades D. The impact of obesity on the outcomes of 1,153 critically injured blunt trauma patients. J Trauma. 2005;59(5):1048-51.

28. Secher NJ, Arnsbo P, Andersen LH, Thomsen A. Measurements of cardiac stroke volume in various body positions in pregnancy and during Caesarean section: a comparison between thermodilution and impedance cardiography. Scand J Clin Lab Invest. 1979;39(6):569-76.

29. Scott DB, Kerr MG. Inferior Vena Caval Pressure in Late Pregnancy. J Obstet Gynaecol Br Commonw. 1963;70:1044-9.

30. Hall JE, Summers RL, Brands MW, Keen H, Alonso-Galicia M. Resistance to metabolic actions of insulin and its role in hypertension. Am J Hypertens. 1994;7(8):772-88.

31. Varvel JR, Donoho DL, Shafer SL. Measuring the predictive performance of computer-controlled infusion pumps. J Pharmacokinet Biopharm. 1992;20(1):63-94.

32. Sargent RG. In: Verification and Validation of Simulation Models. Kuhl ME, Steiger NM, Armstrong FB, Joines JA editors. 2005. 